\title{
Féeries
}

Études sur le conte merveilleux, XVII $-\mathrm{XIX}{ }^{\mathrm{e}}$ siècle

$10 \mid 2013$

Conte et croyance

\section{Jacques Cazotte, La Suite des Mille et Une Nuits}

édition critique établie par Raymonde Robert, Paris, Champion, «Bibliothèque des Génies et des Fées, nº 10 », 1007 p., 2012

\section{Emmanuelle Sempère}

\section{(2) OpenEdition}

12 Journals

Édition électronique

URL : http://journals.openedition.org/feeries/893

DOI : $10.4000 /$ feeries.893

ISSN : 1957-7753

Éditeur

UGA Éditions/Université Grenoble Alpes

\section{Édition imprimée}

Date de publication : 20 septembre 2013

Pagination : 280-286

ISBN : 978-2-84310-253-0

ISSN : 1766-2842

Référence électronique

Emmanuelle Sempère, " Jacques Cazotte, La Suite des Mille et Une Nuits », Féeries [En ligne], 10 | 2013, mis en ligne le 20 mars 2015, consulté le 24 septembre 2020. URL : http://journals.openedition.org/ feeries/893 ; DOI : https://doi.org/10.4000/feeries.893 
avons commentés sont tous le fait d'auteurs qui avaient pris leurs distances par rapport au catholicisme ambiant. Tout se passe comme s'ils y étaient devenus aussitôt profondément étrangers pour ne réussir jamais, quand ils étaient tentés d'y retourner, que des retrouvailles très impropres.» D'une certaine façon, seul Montesquieu aurait permis, bien lu, une approche plus empathique de la religion, et Benjamin Constant l'invoque au seuil de sa longue étude et en comprend la logique pour réfléchir sur la Révolution française, parvenant à en faire une critique en faveur du libéralisme, comme Châteaubriand donc et non comme Burke : «L'homme s'applaudit d'avoir repoussé tous les préjugés, toutes les erreurs, toutes les craintes, et toutes les craintes, tous les préjugés, toutes les erreurs semblent déchaînés. On a proclamé l'empire de la raison, et tout l'univers est frappé de délire; tous les systèmes se fondent sur le calcul, s'adressent à l'intérêt, permettent le plaisir, recommandent le repos, et jamais les égarements ne furent plus honteux, les agitations plus désordonnées, les douleurs plus poignantes» (De la religion, I, I dans Euvres, Paris, Gallimard, "Bibliothèque de la Pléiade», I957, p. 139I). Il appartient à un autre romancier libéral, Walter Scott, d'avoir su entrer dans la conscience fanatique sans la réduire à sa dénonciation indignée et complaisante et tout en la conduisant à une sorte de modération avec son grand roman The Heart of Midlothian (Tales of my landlord, $\mathrm{I} 8 \mathrm{I} 8$ ). Leçons que les $\mathrm{XIX}^{\mathrm{e}}$ et $\mathrm{XX}^{\mathrm{e}}$ siècles n'ont guère écoutées.

Jean-Paul Sermain

La Suite des Mille et Une Nuits, Jacques Cazotte, édition critique établie par Raymonde Robert, Paris, Champion, «Bibliothèque des Génies et des Fées", nº IO, 1007 p., 2012.

La Suite des Mille et Une Nuits constitue le dernier des volumes de "contes orientaux" choisis par les éditions Champion pour leur collection de la «Bibliothèque des Génies et des fées»; c'en est aussi l'œuvre la plus tardive, et l'une des rares à avoir été publiée en tant qu'inédit par Paul Barde, dans les quatre derniers tomes du Cabinet des fées en I788 et I789 - après que le tome 37 avait dressé la liste des ouvrages de sa collection et une notice des auteurs, donnant l'impression d'un achèvement. Avec cette "Suite», également titrée "Continuation» (voir les précisions de R. Robert p. 57), Cazotte signe une œuvre de transition, qui prépare la vague de l'orientalisme du XIX ${ }^{\text {e }}$ siècle : il ne s'agit plus d'une traduction-adaptation, telle que 
les spécialistes de l'Orient comme Galland ou Pétis pouvaient en offrir, ni d'une réécriture irrévérencieuse et parodique à la manière d'Hamilton, de Crébillon ou encore de Voltaire, et ce n'est pas encore la liberté exaltée de l'orientalisme romantique.

Raymonde Robert, qui a déjà signé pour cette collection l'édition critique de certains contes orientaux de Pétis de la Croix et de ceux de l'abbé Bignon ${ }^{\mathrm{I}}$, s'est attelée à la tâche difficile de retrouver les sources d'un auteur qui n'était pas du tout spécialiste de l'Orient et dont on ne sait pas vraiment pourquoi ni comment il a entrepris de prolonger le texte de Galland en s'appuyant sur un manuscrit nouvellement apporté en France par un prêtre syrien, le manuscrit 3637. Comment Cazotte est-il entré en relation avec Al-Káhin Diyánisiás Sháwis, francisé en dom Chavis, "prêtre de la congrégation de Saint Basile qui enseign[ait] la langue arabe au Collège Royal "? Est-ce Paul Barde, éditeur avisé, qui a saisi l'opportunité de bénéficier du succès des Nuits? Et pourquoi Paul Barde aurait-il fait appel à Cazotte, si c'est ainsi que cela s'est passé? L’enquête sur ces questions (p. 8 et suiv.) n'a pas permis, explique R. Robert, de faire toute la lumière sur la genèse de l'œuvre. Reste que La Suite s'inscrit clairement dans un contexte où l'Orient est à la mode, mais aussi dans un moment où la richesse de la structure des Nuits et de leur logique d'accroissement a été perçue par les auteurs comme par le public.

Quant aux motivations de Cazotte et au lien entre cette œuvre tardive et ses autres productions, qu'il s'agisse du Diable amoureux ou des contes parodiques (édités, au volume i6 de la collection, par A. Defrance, et dont est ici reprise la biographie de l'auteur), R. Robert ne cache pas que ni la Correspondance de Cazotte ni la critique du temps ne livrent beaucoup d'informations. Elle exprime la conviction d'une unité profonde de l'œuvre, placée sous le signe du titre choisi par Cazotte à ses œuvres complètes — des " œuvres badines et morales» — et souligne que Cazotte " appartient à son siècle» par "cette capacité à changer de registre et à faire se succéder textes joueurs et légers et textes graves, voire moralisateurs" (p. I7). Ce faisant, R. Robert note cependant une "progression du badin vers le moral» (p. I8) dans le recueil de La Suite et elle l'interprète comme le reflet de l'évolution personnelle d'un auteur de plus en plus attaché à la

I. Ainsi que celle des contes de Mademoiselle Lhéritier, Mademoiselle Bernard, Mademoiselle de La Force, Madame Durand, Madame d'Auneuil («Bibliothèque des Génies et des Fées», vol. 2, 2005), de ceux de Mesdames Levesque, de Gomez, de Dreuillet, Le Marchand, de Lintot, de Lassay, Fagnan, Mademoiselle Falques (Ibid., vol. I3, 2007). Pour Pétis de la Croix et Bignon, voir vol. 8, 2006. 
monarchie de droit divin et à l'église catholique — pour ce second point, le flou qui entoure l'évolution spirituelle de Cazotte ne se trouve pas totalement dissipé. Sur la question du martinisme, il faudrait le regard d'un spécialiste de la question — on peut regretter que, concernant Saint-Martin, seul le texte numérisé des Erreurs et de la vérité soit cité alors qu'il existe de nombreux travaux qui auraient pu éclairer le propos (dont le très clair Louis Claude de Saint-Martin, le philosophe inconnu de N. Jacques-Lefèvre, Dervy, 2003). R. Robert décrit l'écheveau d'inspirations qui entoure par exemple le thème de la révolte des anges après la création du monde : ce motif qui est au cœur de deux des contes de La Suite peut ainsi s'apparenter au martinisme, mais tout aussi bien renvoyer au Livre d'Hénoch (lequel pose aussi la question de la tradition chrétienne ou du martinisme), ou encore à une théorie islamique qui avait intéressé l'abbé Bignon et que ce dernier explique à ses lecteurs dans l'Avertissement de ses Aventures d'Abdalla - sans compter la version (fortement distanciée sinon comique, ajouterons-nous) qu'en délivre Le Comte de Gabalis de Montfaucon de Villars. C'est bien à un «mélange problématique» entre "sérieux, badin et merveilleux ésotérique» (p. 29) que l'œuvre de Cazotte confronte son lecteur. L'examen de la réception de l'œuvre, aux pages 3I-36, confirme cette grande ambivalence. La bibliographie critique (pages 58-60) montre bien l'accélération de l'intérêt pour ce Cazotte tardif; on pourra s'étonner de la partialité des choix qui y sont faits : pour donner un exemple, du colloque «Visages de Cazotte» organisé par F. Gevrey et J.-L. Haquette, seule la contribution de C. Cazenobe est citée - il est vrai que le Cazotte chrétien, voire «franciscain», que ces analyses développent conforte la lecture de R. Robert. Il nous semble utile de préciser que l'inspiration ésotérique a été analysée par de nombreux chercheurs, depuis N. Minerva, et qu'elle n'est pas seulement le lieu d'un leurre. La complexité de l'œuvre - qu'il s'agisse d'ambivalence, d'évolution de l'auteur ou de difficulté pour les lecteurs modernes d'appréhender les délicats rapports entre illuminisme et Lumières - n'est ainsi que partiellement exposée.

Le rapport de La Suite des Mille et Une Nuits au corpus oriental est quant à lui bien explicité. L'œuvre de Cazotte fut présentée par P. Barde comme l'authentique suite du manuscrit incomplet traduit et adapté par Galland, alors que le manuscrit apporté par Chavis n'est pas divisé en nuits et que les contes qu'il contient sont autonomes — il s'agit donc de ces «histoires orphelines» qu'on a ensuite regroupées sous l'appellation de «Nuits» (voir p. Io et I3 de l'édition); cette supercherie a durablement discrédité l'œuvre, et elle a aussi compliqué la tâche des chercheurs qui ont longtemps cherché les sources de Chavis/Cazotte. Les informations données par R. Robert 
s'appuient sur une recherche minutieuse et sur la confrontation des données fournies par les orientalistes Caussin de Perceval et R. Burton au regard et aux connaissances des chercheurs arabisants contemporains, au premier chef Aboubakr Chraïbi (qui a également travaillé avec le chercheur français J.-P. Sermain pour établir et annoter le texte de Galland [GarnierFlammarion, 2004]).

Il s'avère donc que sept des contes choisis et rédigés par Cazotte proviennent du manuscrit 3637 : Le Calife voleur, Le Pouvoir du Destin et l'Histoire d'Halechalbé pour le premier tome, l'Histoire de Simoustapha et l'Histoire de Sinkarib et de ses deux vizirs pour le second, l'Histoire du roi Bohetzad et de ses dix vizirs pour le troisième, enfin l'Histoire d'Habib et de Doratil-Goase qui enjambe sur le quatrième tome. D'autres sources orientales ont été identifiées, mais il ne s'agit jamais, comme pour le manuscrit 3637, d'adaptation directe, soit que Cazotte invente, soit qu'il s'appuie moins sur Chavis que sur d'autres textes de son temps: L'Imbécile ou Histoire de Xaïloun, qui clôt le premier tome, illustre le premier cas, puisque même s'il présente des ressemblances avec le manuscrit 3651, "Hamida the Kaslan», pointé par J. Sadan, la part de création de Cazotte y est, explique R. Robert, prédominante; l'Histoire de Maugraby à la toute fin de l'œuvre illustre le second cas de figure : là encore, une source orientale se laisse reconnaître, le conte égyptien Histoire de Mohammed l'Avisé (qui réfère au conte type 325), mais c'est probablement par des textes français que Cazotte en a eu connaissance. C'est ce qu'explique R. Robert : Cazotte reprend soit le recueil anonyme du Gage touché publié en I70o (mais la référence manque) soit, encore une fois, Les Aventures d'Abdalla de Bignon (voir p. II4).

Les deuxième et quatrième contes du second tome de La Suite, l'Histoire d'Alibengiad sultan d'Hérak et des faux oiseaux de Paradis et l'Histoire de la famille du Schebandad de Surate ne peuvent en l'état des recherches de $\mathrm{R}$. Robert et des spécialistes qu'elle a consultés être rapportées à "aucune source arabe» (voir les notices p. 84 et 9I). Le premier de ces contes est mis, de façon très précise et suggestive, en parallèle avec un autre conte de Cazotte, La Belle par accident, dont on ne connaît pas la date de rédaction; l'analyse est d'autant plus fouillée qu' «il est vraisemblable que [l'Histoire d'Alibengiad] a été entièrement imaginé[e] par Cazotte»(p. 84). L'accent est mis sur la critique du merveilleux et la démystification des croyances - le héros est un fou qui croit au merveilleux. Mais il nous a semblé que le ton en était sensiblement différent; aussi la lecture du passage final — le sultan refusant de prononcer «Au nom du grand Kokopilesobe, seul Dieu de la Terre» affirme qu' «il n'y a qu'un seul Dieu, et Mahomet est son prophète» (p. 424) — comme «changement dans la formule magique» (p. 85) 
pourra-t-elle paraître réductrice. Mais il est vrai que dans ce conte inventé par Cazotte, la mise à distance du merveilleux est frappante; c'est aussi ce que R. Robert met au jour pour l'Histoire de la famille du Schebandad, dans lequel «les baudruches [du merveilleux] sont dégonflées par le biais de ruses très humaines et au moyen d'outils ordinaires" (p. 94). Cazotte met en scène dans ce texte un débat sur la valeur des contes, au travers d'un " concours de contage» (p. 9I) : la fille du Schebandad de Surate, pour départager ses trois prétendants, leur fait raconter un conte en présence de sa "bonne nourrice» (p. 468), Nané, dont la cécité est invoquée comme garantie. Que Cazotte ait plus souvent guerroyé avec Voltaire qu'admis l'influence du philosophe sur son œuvre n'empêchera pas le lecteur moderne de noter que, à l'instar du Sadi de Zadig, l'avisée jeune femme affirme la valeur instructive des contes, à condition qu'ils aient les qualités que la nourrice expose à la fin : loin de ne "rien signifier", le troisième conte tire sa force de son "allégorie ingénieuse». Il s'agit comme le précisent deux notes de $\mathrm{R}$. Robert d'une réaffirmation de la définition classique du conte, comme moyen d'instruire en divertissant, mais, comme chez Voltaire, il s'agit moins ici de morale que de vérité philosophique. En effet, l'allégorie du «rêve de Valid-Hasen» concerne la connaissance, voire la "recherche scientifique» selon R. Robert : en jeu, «les mystères de la nature» (p. 94). L'image du noyer dessine deux démarches : d'une part les «curieux qui font de la besogne mal entendue, en abattant des coquilles vides et des feuilles et en se disputant orgueilleusement le fruit de leurs mauvais travaux", et d'autre part l'" homme plus adroit [...] qui fait tomber la noix du sommet de l'arbre; il l'ouvre et la vérité y est renfermée sous l'emblème d'un million de petits noyers, produits d'un germe unique : on doit cette découverte au hasard, père de toutes celles qui sont utiles» (p. 518). Au commentaire de R. Robert, on peut ajouter que le conte de Cazotte résonne ici clairement avec l'épistémologie des Lumières, chez Diderot ou d'autres plus obscurs, tel Tiphaigne qui, dans Amilec ou la graine d'hommes (éd. P. Vincent, PURH, 20I2), en I754, associe la découverte de la vérité également à un rêve qui efface les errances de l'étude : on se trompe "par réflexion, [on se] désabus[e] par hasard» (ouvr. cité, p. 93).

R. Robert dans ses notices met en avant la démarche poétique de Cazotte, plus créateur qu'adaptateur; très méprisant pour le "mot à mot" de son informateur, Cazotte prend toute liberté pour réinventer les contes. On retiendra, parmi d'autres traits d'écriture analysés par R. Robert, le choix de Cazotte de conserver le lyrisme des poèmes orientaux : contrairement à Galland qui les évacue, comme le souligne R. Robert (p. 72), Cazotte saisit l'opportunité de recomposer en vers mêlés les poèmes que lui transmet 
Chavis (Histoire d'Halechalbé). Même lorsqu'il suit le manuscrit 3637, Cazotte «transforme» (p. 79, par ex.) profondément son matériau : soit qu'il associe plusieurs sources, soit qu'il en modifie la structure, soit qu'il veuille en corriger le message. L'analyse du conte de Simoustapha est particulièrement suggestive; R. Robert y pointe l'effacement de "détails qui sentent par trop la sorcellerie populaire» (p. 79), choix qui sert le «syncrétisme» (p. 8o) entre "christianisme et islam ", tout en s'associant, de façon fort complexe, à une "manipulation de l'ésotérisme» (p. 8I). L'intérêt pour le surnaturel - absent du seul Sinkarib - est un trait commun à toute l'œuvre de Cazotte comme le rappelle R. Robert. La spécificité de son recueil oriental est d'orienter cet intérêt vers un message chrétien. Deux exemples, pris à l'Histoire de Bohetzad : dans le troisième conte, "Le Patient», R. Robert note que Cazotte remplace le motif de la vengeance par l'exécution des "décrets du ciel» (p. 99), ce qui convient autant à l'islam qu'au christianisme; dans le cinquième conte en revanche, les modifications apportées au personnage féminin, "Ravie la Résignée» en font clairement une figure de sainte chrétienne (voir les analyses de R. Robert p. Ior et dans le texte le détail de la prière de Ravie p. 580, faite «les yeux et les mains élevés vers le ciel ", bien loin de la prosternation des musulmans). La clausule de l'Histoire de Bohetzad confirme cette orientation : au sultan qui lui demande "Où avez-vous donc puisé cette foule de maximes et de réflexions judicieuses qui ne peuvent être que le fruit de l'expérience et de l'étude?», le conteur intradiégétique, Aladin, répond : «ce n'est pas moi qui parlais, j'étais inspiré d'en-haut» (p. 653). Le dernier conte de Cazotte, Maugraby, pousse plus loin encore la portée religieuse, conduisant R. Robert à évoquer «un axe qu'on peut dire métaphysique qui renvoie à l'orthodoxie catholique la mieux établie : les forces du mal guettent l'homme en se dissimulant sous des formes trompeuses» (p. II7). Reste que, comme le note par ailleurs R. Robert, de nombreux motifs issus d'autres traditions ne cadrent pas avec cette "orthodoxie catholique» et on ne peut que souhaiter que des chercheurs continuent à les interroger.

Lédition, outre les résumés traditionnels dans la collection, propose un index des principaux personnages qui sera d'une grande aide aux chercheurs pour enquêter sur d'autres recoupements de sources, continuant le nécessaire et passionnant travail concernant le dialogue interculturel que les contes orientaux et/ou orientalisants (c'est-à-dire inventés) donnent à entendre. Il faut cependant mentionner que la facture de l'ouvrage pèche par un assez grand nombre de coquilles laissées dans l'appareil critique, ce qui ne rend pas justice au travail de l'éditeur et rend moins acceptable encore le prix prohibitif des ouvrages de cette collection. 
Reste que R. Robert offre ici aux chercheurs un état très documenté de la double question des intentions et des sources d'une ouvre qui compte parmi les plus déroutantes du corpus orientalisant par ses ambivalences et ses ambiguïtés tonales. Cette édition servira donc aussi bien aux études cazotiennes, encore trop souvent cantonnées au fameux Diable amoureux, qu'aux travaux de plus en plus nombreux sur le conte oriental.

\section{Emmanuelle Sempère}

L'Orient anglais : Connaissances et fictions au XVIII siècle, Claire Gallien, Oxford, Voltaire Foundation (SVEC), 245 p., 2011.

Les études littéraires «orientalistes» contemporaines ont été vigoureusement interpellées, avec d'autres champs disciplinaires, par les thèses d'Edward Said dénonçant l'image inauthentique et mystificatrice des cultures et des réalités orientales diffusée par la culture occidentale selon une approche en dernière instance colonialiste de son objet ${ }^{2}$. Après qu'il a fallu reconnaître dans bien des domaines la pertinence du réquisitoire, certains travaux récents reprennent le sujet à partir de champs disciplinaires précis, en interrogeant les présupposés d'E. Saïd par un examen circonstancié de la réalité des textes et des époques qu'il analyse ${ }^{3}$. C'est le cas de Claire Gallien dans le présent ouvrage, consacré à l'étude des liens entre la littérature "pseudo orientale» ainsi qu'elle la désigne, et les travaux savants sur l'Orient au XVIII ${ }^{\mathrm{e}}$ siècle, en Angleterre. Son enquête déborde donc très en amont de celle d'E. Saïd dont le point de départ est l'expédition d'Égypte française de I798. Sa thèse centrale est d'établir la réciprocité d'influence de l'orientalisme savant et de la littérature de cette époque, et de détruire, ce faisant, une certaine idée du rapport d'objectivité qui mettrait la vérité de l'objet du côté de la science et sa mystification du côté des fictions. Ce faisant, elle revendique (très salutairement selon moi) la nécessité d'une approche contextualisante et historicisée des textes : «le propre de mon étude est en effet d'offrir une interprétation des rapports entre Europe et Orient spécifique au

2. E. Saïd, Orientalism (1978), L'Orientalisme, L'Orient créé par l'Occident, Paris, Seuil, 2003.

3. Voir notamment $S$. Moussa, «Edward Saïd à l'épreuve des voyageurs français en Orient au XIx ${ }^{e}$ siècle", dans KulturPoetik, vol. 6, n I, 2006, p. 96-Io2; G. Bridet, S. Moussa, Ch. Petr (dir.), L'Usage de l'Inde dans les littératures française et européenne (XVIII'-XX siècles), Les Cahiers de la SIELEC, $\mathrm{n}^{\circ}$ 4, Paris/Pondichéry, Éd. Kailash, 2005. Voir également le site du "Séminaire Orientalismes» (D. Combes, D. Lançon, S. Moussa, M. Murat), <http://www.orientalismes.net/>. 